\title{
Need the masses of unstable particles and their antiparticles be equal in the CPT-invariant system?
}

\author{
K. Urbanowski* \\ University of Zielona Gora, Institute of Physics, \\ ul. Podgorna 50, 65-246 Zielona Gora, Poland.
}

October 24, 2018

PACS numbers: 03.65.Ca., 11.30.Er., 11.10.St., 14.40.Aq.

Keywords: CPT invariance; Unstable particles; Particle-antiparticle masses; Matter-antimatter asymmetry.

\begin{abstract}
We show that the real parts of diagonal matrix elements of the exact effective Hamiltonian governing the time evolution in the subspace of states of neutral kaons and similar particles can not be equal for $t>t_{0}\left(t_{0}\right.$ is the instant of creation of the pair $\left.K_{0}, \bar{K}_{0}\right)$ when the total system under consideration is CPT invariant but CP noninvariant. The unusual consequence of this result is that, contrary to the properties of stable particles, the masses of the unstable particle, e.g.. $K_{0}$, and its antiparticle, $\bar{K}_{0}$, need not be equal for $t \gg t_{0}$ in the case of preserved $\mathrm{CPT}$ and violated $\mathrm{CP}$ symmetries.
\end{abstract}

\section{Introduction}

All known CP- and hypothetically possible CPT-violation effects in the neutral kaon complex are described by solving the Schrödinger-like evolution

*e-mail: K.Urbanowski@if.uz.zgora.pl; K.Urbanowski@proton.if.uz.zgora.pl 
equation [1] - 11] (we use $\hbar=c=1$ units)

$$
i \frac{\partial}{\partial t}\left|\psi ; t>_{\|}=H_{\|}\right| \psi ; t>_{\|}, \quad\left(t \geq t_{0}\right)
$$

for $\mid \psi ; t>_{\|}$belonging to the subspace $\mathcal{H}_{\|} \subset \mathcal{H}$ (where $\mathcal{H}$ is the state space of the physical system under investigation), e.g., spanned by orthonormal neutral kaons states $\left|K_{0}>,\right| \bar{K}_{0}>$, and so on, (then states corresponding with the decay products belong to $\mathcal{H} \ominus \mathcal{H}_{\|} \stackrel{\text { def }}{=} \mathcal{H}_{\perp}$ ), and nonhermitian effective Hamiltonian $H_{\|}$obtained usually by means of the Lee-Oehme-Yang (LOY) approach (within the Weisskopf-Wigner approximation (WW)) [1] — 11]:

$$
H_{\|} \equiv M-\frac{i}{2} \Gamma
$$

where $M=M^{+}, \Gamma=\Gamma^{+}$are $(2 \times 2)$ matrices. In a general case $H_{\|}$can depend on time $t, H_{\|} \equiv H_{\|}(t)$, [12, 13, 14. The effective Hamiltonians $H_{\|}$ are usually derived by rewriting the Schödinger equation in the kaon rest frame:

$$
i \frac{\partial}{\partial t} U(t)\left|\psi>_{\|}=H U(t)\right| \psi>_{\|}, \quad U\left(t=t_{0}\right)=I,
$$

where $I$ is the unit operator in $\mathcal{H}, H$ is be total (selfadjoint) Hamiltonian acting in $\mathcal{H}$ and $U(t)$ the total unitary evolution operator, $\mid \psi>_{\|} \equiv$ $\mid \psi, t=t_{0}>_{\|} \in \mathcal{H}_{\|}$is the initial state of the system.

Solutions of Eq. (11) can be written in matrix form and such a matrix defines the evolution operator (which is usually nonunitary) $U_{\|}(t)$ acting in $\mathcal{H}_{\|}:$

$$
\left|\psi ; t>_{\|} \stackrel{\text { def }}{=} U_{\|}(t)\right| \psi>_{\|}
$$

where,

$$
\left|\psi>_{\|} \equiv q_{1}\right| \mathbf{1}>+q_{2} \mid \mathbf{2}>,
$$

and $\mid \mathbf{1}>$ stands for the vectors of the $\left|K_{0}>,\right| B_{0}>$ type and $\mid \mathbf{2}>$ denotes antiparticles of the particle "1": $\left|\bar{K}_{0}>, \bar{B}_{0}>,<\mathbf{j}\right| \mathbf{k}>=\delta_{j k}, j, k=1,2$.

Relations between matrix elements of $H_{\|}$implied by the CPT-transformation properties of the Hamiltonian $H$ of the total system, containing the neutral kaon complex as a subsystem, are crucial for designing CPTinvariance and $\mathrm{CP}$-violation tests and for proper interpretation of their results. The standard interpretation of matrix elements, $h_{j k}=<\mathbf{j}\left|H_{\|}\right| \mathbf{k}>$, $(j . k=1,2)$, of the effective Hamiltonian $H_{\|}$follows form the properties of 
the LOY effective Hamiltonian $H_{\|} \equiv H_{L O Y}$. In many papers it is assumed that the real parts, $\Re($.$) , of the diagonal matrix elements of H_{\|}$:

$$
\Re\left(h_{j j}\right) \equiv M_{j j}, \quad(j=1,2),
$$

where

$$
h_{j k}=<\mathbf{j}\left|H_{\|}\right| \mathbf{k}>,(j, k=1,2),
$$

correspond to the masses of particle "1" and its antiparticle "2" respectively [1] - 11], (and such an interpretation of $\Re\left(h_{11}\right)$ and $\Re\left(h_{22}\right)$ will be used in this paper), whereas the imaginary parts, $\Im($.$) , are interpreted as the decay$ widths of these particles, $\Gamma_{j j} \equiv-2 \Im\left(h_{j j}\right),(j=1,2),[1]$ [1], 15].

Taking $H_{\|}=H_{L O Y}$ and assuming that the CPT invariance holds in the system considered one easily finds the standard result of the LOY approach

$$
h_{11}^{L O Y}=h_{22}^{L O Y}
$$

which, among others, means that

$$
M_{11}^{L O Y}=M_{22}^{L O Y},
$$

where $M_{j j}^{L O Y}=\Re\left(h_{j j}^{L O Y}\right)$ and $h_{j j}^{L O Y}=<\mathbf{j}\left|H_{L O Y}\right| \mathbf{j}>,(j=1,2)$. This last relation is interpreted as the equality of masses of the unstable particle $|\mathbf{1}\rangle$ and its antiparticle $\mid \mathbf{2}>$.

In [16] using the Khalfin's Theorem [9, 17] - 19] it was proved that, contrary to the conclusion drawn in (8), the diagonal matrix elements of the exact effective Hamiltonian $H_{\|}$in a CPT invariant but $\mathrm{CP}$ noninvariant system must be different for $t>t_{0}$. This proof is rigorous. A crucial conclusion following from this property was put forward in [16. This conclusion states that, contrary to property (9), the masses of unstable particle and its antiparticle should be different for $t>t_{0}$. In fact, this conclusion was not supported there by a direct and rigorous proof. The aim of this note is to complete the result of [16] and to prove this conclusion. Strictly speaking, to prove rigorously that in the case of the exact $H_{\|}$it must be $\Re\left(h_{11}-h_{22}\right) \neq 0$ for $t>t_{0}$, if the CPT symmetry holds and CP is violated. In order to realize this purpose, the method applied in [14] will be used. 


\section{CPT transformation properties of the exact $H_{\|}$}

The aim of this Section is to show that in the case of the exact $H_{\|}$, the standard LOY relation (9) does not occur if the total system under consideration is CPT invariant, $\Theta H \Theta^{-1}=H^{+} \equiv H$, that is

$$
[\Theta, H]=0
$$

where $\Theta$ is the antiunitary operator:

$$
\Theta \stackrel{\text { def }}{=} \mathcal{C P} \mathcal{T}
$$

and CP noninvariant.

Let $P$ denote the projection operator onto the subspace $\mathcal{H}_{\|}$:

$$
P \mathcal{H}=\mathcal{H}_{\|}, \quad P=P^{2}=P^{+},
$$

then the subspace of decay products $\mathcal{H}_{\perp}$ equals

$$
\mathcal{H}_{\perp}=(I-P) \mathcal{H} \stackrel{\text { def }}{=} Q \mathcal{H}, \quad Q \equiv I-P .
$$

For the case of neutral kaons or neutral $B$-mesons, etc., the projector $P$ can be chosen as follows:

$$
P \equiv|\mathbf{1}><\mathbf{1}|+|\mathbf{2}><\mathbf{2}|
$$

We assume that time independent basis vectors $\mid K_{0}>$ and $\mid \bar{K}_{0}>$ are defined analogously to corresponding vectors used in the LOY theory of time evolution in the neutral kaon complex [1] - [1]: Vectors $\mid K_{0}>$ and $\mid \bar{K}_{0}>$ can be identified with the eigenvectors of the so-called free Hamiltonian $H^{(0)} \equiv H_{\text {strong }}=H-H^{(1)}$, where $H^{(1)} \equiv H_{\text {int }}$ denotes weak and other interactions which are responsible for transitions between eigenvectors of $H^{(0)}$, i.e., for the decay process. This means that

$$
\left[P, H^{(0)}\right]=0 .
$$

The condition guaranteeing the occurrence of transitions between subspaces $\mathcal{H}_{\|}$and $\mathcal{H}_{\perp}$, i.e., a decay process of states in $\mathcal{H}_{\|}$, can be written as follows

$$
[P, H] \neq 0
$$


Note that Eq. (3) means that $U(t)=\exp (-i t H)$. Now, knowing $U(t)$, the exact evolution operator $U_{\|}(t)$ (4) for $\mathcal{H}_{\|}$can be expressed using the projector $P$ as follows

$$
U_{\|}(t) \equiv P U(t) P .
$$

We have $U_{\|}(0) \equiv P$. In [12, 13, 14 an observation was made that for every effective Hamiltonian $H_{\|}$governing the time evolution in subspace $\mathcal{H}_{\|} \equiv P \mathcal{H}$, the following identity holds:

$$
H_{\|} \equiv H_{\|}(t) \equiv i \frac{\partial U_{\|}(t)}{\partial t}\left[U_{\|}(t)\right]^{-1},
$$

where the operator $\left[U_{\|}(t)\right]^{-1}$ is defined as follows [16]

$$
\left[U_{\|}(t)\right]^{-1} U_{\|}(t)=U_{\|}(t)\left[U_{\|}(t)\right]^{-1} \equiv P .
$$

(These last two relations were also used in [16]). It can be easily verified that $H_{\|} \equiv H_{L O Y}$, fulfills the identity (18).

In the nontrivial case (16) from (18) (see Appendix, formula (A.2)), using (3) and (17) we find

$$
\begin{aligned}
H_{\|}(t) & \equiv P H U(t) P\left[U_{\|}(t)\right]^{-1} P \\
& \equiv P H P+P H Q U(t)\left[U_{\|}(t)\right]^{-1} P \\
& \stackrel{\text { def }}{=} P H P+V_{\|}(t) .
\end{aligned}
$$

Thus [20, 21, 22, 23]

$$
H_{\|}(0) \equiv P H P, \quad V_{\|}(0)=0, \quad V_{\|}(t \rightarrow 0) \simeq-i t P H Q H P,
$$

so, in general $H_{\|}(0) \neq H_{\|}\left(t \gg t_{0}=0\right)\left[20,21\right.$, 22] and $V_{\|}(t \neq 0) \neq V_{\|}^{+}(t \neq 0)$, $H_{\|}(t \neq 0) \neq H_{\|}^{+}(t \neq 0)$.

Now let us pass on to the investigation of the CPT-transformation properties of $H_{\|}$. We assume that vectors $|\mathbf{1}>,| \mathbf{2}>$ are related to each other through the transformation:

$$
\Theta|\mathbf{1}>\stackrel{\text { def }}{=}-| \mathbf{2}>, \quad \Theta|\mathbf{2}>\stackrel{\text { def }}{=}-| \mathbf{1}>,
$$

Besides, there is only one assumption for the anti-linear operator $\Theta$ (11) describing the CPT-transformation in $\mathcal{H}$. We require CPT-invariance of 
$\mathcal{H}_{\|}$. This means that for the projector $P$ defining this subspace the following relation must hold,

$$
[\Theta, P]=0 .
$$

Using assumption (25) and the identity (20), after some algebra, one finds 24] (see Appendix A)

$$
\left[\Theta, H_{\|}(t)\right]=\mathcal{A}(t)+\mathcal{B}(t)
$$

where:

$$
\begin{aligned}
\mathcal{A}(t) & =P[\Theta, H] U(t) P\left(U_{\|}(t)\right)^{-1} \\
\mathcal{B}(t) & =\left\{P H-P H U(t) P\left(U_{\|}(t)\right)^{-1} P\right\}[\Theta, U(t)] P\left(U_{\|}(t)\right)^{-1} \\
& \equiv\left\{P H-H_{\|}(t) P\right\}[\Theta, U(t)] P\left(U_{\|}(t)\right)^{-1} \\
& \equiv\left\{P H Q-V_{\|}(t) P\right\}[\Theta, U(t)] P\left(U_{\|}(t)\right)^{-1}
\end{aligned}
$$

We observe that for $t=0$

$$
\mathcal{A}(0) \equiv P[\Theta, H] P \text { and } \mathcal{B}(0) \equiv 0
$$

From definitions and the general properties of operators $\mathcal{C}, \mathcal{P}$ and $\mathcal{T}$, 8 , 25. 26. 27] it is known that $\mathcal{T} U(t \neq 0)=U^{+}(t \neq 0) \mathcal{T} \neq U(t \neq 0) \mathcal{T}$ (Wigner's definition for $\mathcal{T}$ is used), and thereby $\Theta U(t \neq 0)=U^{+}(t \neq 0) \Theta$ [25, 26, 27] i.e. $[\Theta, U(t \neq 0)] \neq 0$. So, the component $\mathcal{B}(t)$ in (26) is nonzero for $t \neq 0$ and it is obvious that there is a chance for the $\Theta$-operator to commute with the effective Hamiltonian $H_{\|}(t \neq 0)$ only if $[\Theta, H] \neq 0$. On the other hand, the property $[\Theta, H] \neq 0$ does not imply that $\left[\Theta, H_{\|}(0)\right]=0$ or $\left[\Theta, H_{\|}(0)\right] \neq 0$. These two possibilities are admissible, but if $[\Theta, H]=0$ then there is only one possibility: $\left[\Theta, H_{\|}(0)\right]=0$ [14].

From (26) we find

$$
\Theta H_{\|}(t) \Theta^{-1}-H_{\|}(t) \equiv(\mathcal{A}(t)+\mathcal{B}(t)) \Theta^{-1}
$$

Now, keeping in mind that $|\mathbf{2}>\equiv| \bar{K}_{0}>$ is the antiparticle for $|\mathbf{1}>\equiv| K_{0}>$ and that, by definition, the (anti-unitary) $\Theta$-operator transforms $\mid \mathbf{1}>$ in $|2\rangle[2$ [ 8] according to formulae (24), and $\langle\psi \mid \varphi\rangle=\langle\Theta \varphi \mid \Theta \psi\rangle$, we obtain from (32) (see Appendix A)

$$
h_{11}(t)^{*}-h_{22}(t)=<\mathbf{2}\left|(\mathcal{A}(t)+\mathcal{B}(t)) \Theta^{-1}\right| \mathbf{2}>,
$$


Adding expression (33) to its complex conjugate one gets

$$
\Re\left(h_{11}(t)-h_{22}(t)\right)=\Re<\mathbf{2}\left|(\mathcal{A}(t)+\mathcal{B}(t)) \Theta^{-1}\right| \mathbf{2}>.
$$

Note that if the requirement (16) for the projector $P$ (14) is replaced by the following one:

$$
[P, H]=0,
$$

i.e. if only stationary states are considered instead of unstable states, then one immediately obtains from (27) - (30):

$$
\begin{aligned}
\mathcal{A}(t) & =P[\Theta, H] P, \\
\mathcal{B}(t) & =0 .
\end{aligned}
$$

Let us assume that conditions (16) and (10) hold. For the stationary states (35), the assumption (101), relations (36), (37) and (34) yield $\Re\left(h_{11}(t)-h_{22}(t)\right)=0$.

Now let us consider the case of unstable states, i.e., states $|\mathbf{1}>,| \mathbf{2}>$, which lead to such projection operator $P$ (14) that condition (16) holds. If in this case (10) also holds then $\mathcal{A}(t=0) \equiv 0$ (see (31)) and thus $\left[\Theta, H_{\|}(0)\right]$ $=0$, which is in agreement with earlier, similar results [14, 16]. This means that at $t=0$ :

$$
\Re\left(h_{11}(0)-h_{22}(0)\right)=H_{11}-H_{22}=0,
$$

where

$$
H_{j k}=<\mathbf{j}|H| \mathbf{k}>, \quad(j, k=1,2) .
$$

Let $t>0$. In this case we have $\Theta U(t)=U^{+}(t) \Theta$, which gives $\Theta U_{\|}(t)=$ $U_{\|}^{+}(t) \Theta, \Theta U_{\|}^{-1}(t)=\left(U_{\|}^{+}(t)\right)^{-1} \Theta$, and

$$
[\Theta, U(t)]=-2 i(\Im U(t)) \Theta
$$

This relation leads to the following result in the case of conserved CPTsymmetry

$$
\begin{aligned}
\mathcal{B}(t) & =-2 i P\left\{H-H_{\|}(t) P\right\}(\Im U(t)) P\left(U_{\|}^{+}(t)\right)^{-1} \Theta \\
& \equiv-2 i\left\{P H Q-V_{\|}(t) P\right\}(\Im U(t)) P\left(U_{\|}^{+}(t)\right)^{-1} \Theta,
\end{aligned}
$$

which means that generally, in any case $\mathcal{B}(t>0) \neq 0$.

Formulae (41), (42) allow us to conclude that $<\mathbf{2}\left|\mathcal{B}(0) \Theta^{-1}\right| \mathbf{2}>=0$ and $\Re<\mathbf{2}\left|\mathcal{B}(t>0) \Theta^{-1}\right| \mathbf{2}>\neq 0$, if condition (10) holds. This means that in this 
case it must be $\Re\left(h_{11}(t)\right) \neq \Re\left(h_{22}(t)\right)$ for $t>0$. So, there is no possibility for $\Re\left(h_{11}\right)$ to equal $\Re\left(h_{22}\right)$ for $t>0$ in the considered case of $P$ fulfilling the condition (16) (i.e., for unstable states) when CPT-symmetry is conserved.

Using identity (20) and assuming that $[\mathcal{C P}, H]=0$ and (10) hold it is not difficult to show that in such case $h_{11}(t)=h_{22}(t)$.

\section{Discussion.}

All the above considerations lead to the following conclusions for the matrix elements $h_{j k}$ of the exact effective Hamiltonian $H_{\|}$governing the time evolution in neutral kaons subspace:

\section{Conclusion 1}

If $[\Theta, H]=0$ and $[\mathcal{C P}, H] \neq 0$ then it follows that $M_{11} \equiv \Re\left(h_{11}(t>0)\right)$ $\neq \Re\left(h_{22}(t>0)\right) \equiv M_{22}$, that is that the mass of the unstable particle " 1 " must be different from the mass of its antiparticle " 2 " for $t>t_{0}=0$.

One should remember that the above conclusion derived from relation (34) concerns only the real parts of $h_{11}(t>0)$ and $h_{22}(t>0)$ and it is in excellent agreement with the results presented in [16]. Relations (32) - (34) give us no information about the imaginary parts of $h_{11}$ and $h_{22}$. One cannot infer from (34) that $[\Theta, H]=0$ follows $\Im\left(h_{11}\right) \neq \Im\left(h_{22}\right)$. The case when $[\Theta, H]=0$ follows $\Re\left(h_{11}(t>0)\right) \neq \Re\left(h_{22}(t>0)\right)$ and $\Im\left(h_{11}\right)=\Im\left(h_{22}\right)$, is not in conflict with relations (32) - (34). The equality of $\Im\left(h_{11}\right)$ and $\Im\left(h_{22}\right)$ need not imply the equality of $\Re\left(h_{11}\right)$ and $\Re\left(h_{22}\right)$ and vice versa. This means that the Bell-Steinberger relations [28, do not contradict relations (32) (34) and Conclusion 1 following from them. Namely, Bell and Steinberger formulae lead to the equality of $\Im\left(h_{11}\right)$ and $\Im\left(h_{22}\right)$ in the case of conserved CPT-symmetry and do not concern the real parts of the diagonal matrix elements of $H_{\|}$or give any relations between them.

The real parts of the diagonal matrix elements of the mass matrix $H_{\|}$, $h_{11}$ and $h_{22}$, are considered in the literature as masses of unstable particles $|\mathbf{1}>,| \mathbf{2}>$ (e.g., mesons $K_{0}$ and $\bar{K}_{0}$ ). Such an interpretation follows also from properties, (18), (22) and (23) of the exact $H_{\|}(t)$. The interpretation of the diagonal matrix elements of $H_{\|}(t=0)$ is obvious (see (23) ). They have the dimension of the energy (that is, the mass) and $h_{j j}(0) \equiv<\mathbf{j}|H| \mathbf{j}>$, 
$(j=1,2)$. So their interpretation as the masses of the particle "1" and its antiparticle "2" at the instant $t=0$ seems to be explained. Note that from the identity (18) it follows that the exact effective Hamiltonian $H_{\|}(t)$ is a continuous function of time $t$. Therefore the dimension of $H_{\|}(t)$ as the physical quantity at any $t>0$ continues to be the same as that at $t=0$.

From (22) one finds

$$
h_{j k}(t)=H_{j k}+v_{j k}(t), \quad(j, k=1,2),
$$

where $v_{j k}(t)=<\mathbf{j}\left|V_{\|}(t)\right| \mathbf{k}>$. So, at $t>t_{0}=0$ the initial mass (energy) of the particle $j, \Re\left(h_{j j}(0)\right)=<\mathbf{j}|H| \mathbf{j}>\equiv H_{j j}$, in the state $\mid \mathbf{j}>$, shifts and takes the value

$$
\Re\left(h_{j j}(t)\right)=H_{j j}+\Re\left(v_{j j}(t)\right) .
$$

Every experiment performed at this instant $t$ will indicate the quantity (44) as the energy (i.e., as the mass) of the particle $j$ at time $t$. In the case of neutral particles there are no methods allowing one to differentiate the contribution of $\Re\left(h_{j j}(0)\right)=H_{j j}$ into $\Re\left(h_{j j}(t)\right)$ from the contribution of the shift $\Re\left(v_{j j}(t)\right)$ by means of the measurement performed at the instant $t>0$. The particle $j$ always interacts with the environment at the instant $t$ as the particle with the energy $\Re\left(h_{j j}(t)\right)$. So from the point of view of the relativistic quantum theory the interpretation of $\Re\left(h_{j j}(t)\right)$ as the mass of the particle $j$ seems to be acceptable.

There is another one reason for the adoption of LOY interpretation of the matrix elements of $H_{\|}$in this letter. Note that, as it was mentioned in Sec. 2, the LOY effective Hamiltonian, $H_{L O Y}$ fulfils the identity (18). It seems that the interpretation of matrix elements of any effective Hamiltonian fulfilling this identity can not differ from the interpretation of matrix elements of $H_{L O Y}$.

So Conclusion 1 means that masses of a decaying particle "1" and its antiparticle "2" should be different if the CPT-symmetry is conserved in the system containing these unstable particles. In other words, in the exact theory unstable states $|\mathbf{1}>,| \mathbf{2}>$ appear to be nondegenerate in mass for $t>t_{0}$ if the CPT-symmetry holds and the CP-symmetry does not, in the total system considered. At the same time, relations (35) - (37) and (10) suggest that in the CPT-invariant system masses of a given particle and its aniparticle are equal (i.e., appear to be degenerate) only in the case of stationary (stable) states $|\mathbf{1}>,| \mathbf{2}>$. The case, when vectors $|\mathbf{1}>,| \mathbf{2}>$ describe pairs of particles $p, \bar{p}$, or $e^{-}, e^{+}$, can be considered as an example of 
such states. All these conclusions contradict the standard result of the LOY and related approaches.

Results of the previous Section and Conclusions 1 are not in conflict with such implications of the CPT-invariance as the equality of particle and antiparticle decay rates - see [16]. On the other hand the consequences (8) and (9) of the LOY theory are in conflict with the results of Sec. 2 and Conclusion 1 obtained without approximations but they are in agreement with the rigorous result obtained in [16].

Note that in fact the above conclusions about the masses of unstable particles under consideration are not in conflict with the rigorous and consistent treatment of quantum theory. From (10) (or from the CPT Theorem [29]) it only follows that the masses of particle and antiparticle eigenstates of $H$ (i.e., masses of stationary states for $H$ ) should be the same in the CPT invariant system - see [16]. Such a conclusion can not be derived from (10) for particle $\mid \mathbf{1}>$ and its antiparticle $\mid \mathbf{2}>$ if they are unstable, i.e., if states $|\mathbf{1}>,| \mathbf{2}>$ are not eigenstates of $H$. Note also that the proof of the CPT Theorem makes use of the properties of asymptotic states [29]. Such states do not exist for unstable particles. What is more, one should remember that the CPT Theorem of axiomatic quantum field theory has been proved for quantum fields corresponding to stable quantum objects and only such fields are considered in the axiomatic quantum field theory. There is no axiomatic quantum field theory of unstable quantum particles. So, all implications of the CPT Theorem (including those obtained within the S-matrix method) need not be valid for decaying particles prepared at some initial instant $t_{0}=0$ and then evolving in time $t \geq 0$. Simply, the consequences of CPT invariance need not be the same for systems in which time $t$ varies from $t=-\infty$ to $t=+\infty$ and for systems in which $t$ can vary only from $t=t_{0}>-\infty$ to $t=+\infty$. Similar doubts about the fundamental nature of the CPT Theorem were formulated in [30, where the applicability of this theorem for QCD was considered. One should also remember that conclusions about the equality of masses of stable particles and their antiparticles following from the properties of the S-matrix can not be extrapolated to the case of unstable states. Simply, there is no S-matrix for unstable states.

The important consequence of Conclusion 1 is that the conventional interpretation of the tests, which are sensitive to the difference $\Re\left(h_{11}-h_{22}\right)$, as the CPT invariance test in neutral kaon complex, need not be longer valid. An example of a such test is considered in [31.

Another consequence of the main result of the Section 2, that is of the 
Conclusion 1 concerns properties of the scalar product of eigenvectors $\mid l>$, $\mid s>$ of $H_{\|}$,

$$
H_{\|}\left|l(s)>=\mu_{l(s)}\right| l(s)>.
$$

for the eigenvalues $\mu_{l(s)}=\frac{1}{2}\left(h_{11}+h_{22}\right)-(+) \frac{1}{2}\left[\left(h_{11}-h_{22}\right)^{2}+4 h_{12} h_{21}\right]^{1 / 2} \equiv$ $m_{l(s)}-\frac{i}{2} \gamma_{l(s)}$, where $m_{l(s)}, \gamma_{l(s)}$ are real. These eigenvectors correspond to the long (the vector $\mid l>$ ) and short (the vector $\mid s>$ ) living superpositions of $K_{0}$ and $\overline{K_{0}}$.

Using the eigenvectors

$$
\mid K_{1(2)}>\stackrel{\text { def }}{=} 2^{-1 / 2}(|\mathbf{1}>+(-)| \mathbf{2}>),
$$

of the $\mathrm{CP}$-transformation for the eigenvalues \pm 1 (we define $\mathcal{C P}|\mathbf{1}>=-| \mathbf{2}>$, $\mathcal{C P}|\mathbf{2}>=-| \mathbf{1}>)$, vectors $\mid l>$ and $\mid s>$ can be expressed as follows

$$
\mid l(s)>\equiv\left(1+\left|\varepsilon_{l(s)}\right|^{2}\right)^{-1 / 2}\left[\left|K_{2(1)}>+\varepsilon_{l(s)}\right| K_{1(2)}>\right] .
$$

This last relation leads to the following formula for the product $\langle s \mid l\rangle$,

$$
<s \mid l>\equiv N\left(\varepsilon_{s}^{*}+\varepsilon_{l}^{*}\right),
$$

where $N=N^{*}=\left[\left(1+\left|\varepsilon_{s}\right|^{2}\right)\left(1+\left|\varepsilon_{l}\right|^{2}\right)\right]^{-1 / 2}$. By means of the following parameters

$$
\begin{gathered}
\varepsilon \stackrel{\text { def }}{=} \frac{1}{2}\left(\varepsilon_{s}+\varepsilon_{l}\right), \\
\delta \stackrel{\text { def }}{=} \frac{1}{2}\left(\varepsilon_{s}-\varepsilon_{l}\right),
\end{gathered}
$$

which are usually are used to describe the scale of $\mathrm{CP}$ - and possible CPT violation effects [3, 4, 6, 10, 15, product (48) can be expressed as follows

$$
<s \mid l>\equiv 2 N(\Re \varepsilon-i \Im \delta) .
$$

There is

$$
\delta \simeq \frac{h_{11}-h_{22}}{2\left(\mu_{s}-\mu_{l}\right)} \equiv \delta_{\|} e^{i \phi_{S W}}+\delta_{\perp} e^{i\left(\phi_{S W}+\pi / 2\right)},
$$

in the case of $\left|\varepsilon_{s}\right| \ll 1$ and $\left|\varepsilon_{l}\right| \ll 1$ (see, eg. [15], p. 560). Here $\phi_{S W}$ is the superweak phase, $\tan \phi_{S W}=\frac{2\left(m_{l}-m s\right)}{\gamma_{s}-\gamma_{l}}$, and

$$
\begin{aligned}
\delta_{\|} & =\frac{1}{4} \frac{\Gamma_{11}-\Gamma_{22}}{\sqrt{\left(m_{s}-m_{l}\right)^{2}+\frac{1}{4}\left(\gamma_{s}-\gamma_{l}\right)^{2}}}, \\
\delta_{\perp} & =\frac{1}{2} \frac{\Re\left(h_{11}-h_{22}\right)}{\sqrt{\left(m_{s}-m_{l}\right)^{2}+\frac{1}{4}\left(\gamma_{s}-\gamma_{l}\right)^{2}}},
\end{aligned}
$$


are the real parameters. Thus

$$
\Im \delta=\delta_{\|} \sin \phi_{S W}+\delta_{\perp} \cos \phi_{S W}
$$

The consequence of (8), (9) is that in CPT invariant but CP noninvariant system $\delta_{\|}=\delta_{\|}^{L O Y}=0$ and $\delta_{\perp}=\delta_{\perp}^{L O Y}=0$ which leads to the standard result $\Im \delta^{L O Y}=0$ (here $\delta^{L O Y}$ denotes the parameter $\delta$, (52), calculated for $\left.H_{\|}=H_{L O Y}\right)$. From this property and (51) the conclusion that the product $\langle s| l>$ must be real is drawn in the literature. This conclusion is considered as the standard result. Note that in the light of the main result of Sec. 2 and Conclusion 1 such a conclusion seems to be wrong in the case of the exact effective Hamiltonian $H_{\|}$, that is, in the case of the exact theory. From Conclusion 1 one infers that there must be $\delta_{\perp} \neq 0$, (54) in the case of CPT invariant but $\mathrm{CP}$ noninvariant system and therefore it must be $\Im \delta \neq 0$ (see (55) in such a system. This means that the right hand side of the relation (51) is a complex number and therefore in the case of conserved CPT- and violated $\mathrm{CP}$-symetries, in contrast with the standard result, there must be $<s|l>\neq<s| l>^{*}$ in the real systems.

Properties of the real systems discussed above and described in Conclusion 1 are unobservable for the LOY approximation. In order to obtain at least an estimation of the effects described in these Conclusions, the matrix elements of $H_{\|}$should be calculated much more exactly than it is possible within the LOY theory. A proposal of a more exact approximation is given in 21, 22, 32, 33. This approximation is based on the Krolikowski-Rzewuski equation for a distinguished component of the state vector 34]. All CP - and CPT - transformation properties of the effective Hamiltonian $H_{\|}$calculated within this approximation are consistent with similar properties of the exact effective Hamiltonian and with the result obtained in this paper and [16] .

Within the mentioned more accurate approximation one finds for diagonal matrix elements of $H_{\|} \simeq H_{\|}^{(1)} \stackrel{\text { def }}{=} \lim _{t \rightarrow \infty} H_{\|}^{(1)}(t)$ that the CPT-invariant system in contradistinction to the property (8) obtained within the LOY theory [20, 21, 22, 33]

$$
h_{11} \neq h_{22}
$$

and

$$
h_{11}(t=0)=H_{11} \equiv H_{22}=h_{22}(t=0) .
$$

The relation (57) is consistent with the properties (23) and (38) and the result obtained in [16]. 
Assuming that

$$
\left|H_{12}\right| \ll\left|H_{0}\right|
$$

where $H_{0} \stackrel{\text { def }}{=} \frac{1}{2}\left(H_{11}+H_{22}\right),\left(H_{0}=H_{11} \equiv H_{22}\right.$ if (10) holds), one finds within the mentioned more accurate approximation that (see 31] and (77) in [22])

$$
\Delta h \stackrel{\text { def }}{=} h_{11}-\left.h_{22} \simeq H_{12} \frac{\partial \Sigma_{21}(x)}{\partial x}\right|_{x=H_{0}}-\left.H_{21} \frac{\partial \Sigma_{12}(x)}{\partial x}\right|_{x=H_{0}} \neq 0
$$

Here

$$
\Sigma(\epsilon)=P H Q \frac{1}{Q H Q-\epsilon-i 0} Q H P .
$$

and $\Sigma_{j k}(\epsilon)=<\mathbf{j}|\Sigma(\epsilon)| \mathbf{k}>$.

From the result (59) it follows that $\Delta h=0$ can be achieved only if $H_{12}=$ $H_{21}=0$. Relation (15) implies that $H_{12} \equiv<\mathbf{1}\left|H_{\text {int }}\right| \mathbf{2}>$. If $|\mathbf{1}>\equiv| K_{0}>$ and $|\mathbf{2}>\equiv| \bar{K}_{0}>$ then the strangeness $S$ of the particle "1" equals $S=+1$ while that of " 2 " is $S=-1$. Therefore the interpretation of the hypothetical property $<\mathbf{1}\left|H_{\text {int }}\right| \mathbf{2}>\neq 0$, which can be met in the literature, is that the first order $|\Delta S|=2$ transitions are allowed [5, 6, 7].

So, the property $H_{12}=H_{21}=0$ means that if the first order $|\Delta S|=2$ transitions are forbidden in the $K_{0}, \bar{K}_{0}$ complex then predictions following from the use of the mentioned more accurate approximation and from the LOY theory should lead to the the same masses for $K_{0}$ and for $\bar{K}_{0}$. This does not contradict the Conclusion 1 following from the results of Sec. 2 derived for the exact $H_{\|}$or the rigorous result of [16]: the mass difference is very, very small and should arise at higher orders of this more accurate approximation.

On the other hand from (59) it follows that in the considered approximation $\Delta h \neq 0$ if and only if $H_{12} \equiv<\mathbf{1}\left|H_{\text {int }}\right| \mathbf{2}>\neq 0$. This means that if measurable deviations from the LOY predictions concerning the equality of masses of, e.g. $K_{0}, \bar{K}_{0}$ mesons are ever detected in some tests, then the most plausible interpretation of this result will be the existence of interactions allowing the first order $|\Delta S|=2$ transitions in the system considered [31].

Within the use of the toy Fridrichs-Lee model [9, 21] the following estimation was found in [16]:

$$
\frac{\Re(\Delta h)}{\Re\left(h_{11}+h_{22}\right)} \sim 9,25 \times 10^{-18}\left(\Im\left(<\mathbf{1}\left|H_{\text {int }}\right| \mathbf{2}>\right)\right)[\mathrm{MeV}]^{-1}
$$


This and the estimation $\frac{\left|m_{K_{0}}-m_{\bar{K}_{0}}\right|}{m_{K-\text { average }}}<10^{-18}$ (where $m_{\alpha},\left(\alpha=K_{0}, \bar{K}_{0}\right)$ denotes masses of $K_{0}$ and $\bar{K}_{0}$-meson respectivelly), which can be found in [15], show that possible deviations from the standard picture, that is, from the LOY predictions are much too small to be observed with the present experiments.

Confronting relations (8) with (34), one should remember that, in fact, $H_{L O Y}$ can be considered as the lowest, nontrivial order approximation in the perturbation $H^{(1)}$ : All the terms to higher orders than $\left(H^{(1)}\right)^{2}$ are neglected in $H_{L O Y}$ [1] - 11]. It is obvious that CPT- and other transformation properties of such an approximate effective Hamiltonian and of the exact one need not be the same. Taking into account all the above, it seems that for the proper understanding of the CPT-invariance tests and CPT-invariance, or possible CPT-violation phenomena it is necessary to consider higher order contributions into the LOY-type effective Hamiltonian than those contained in $H_{L O Y}$ or to use a more accurate approximation than LOY.

The result (8) of the LOY approximation is model independent whereas, within the mentioned more accurate approximation, the magnitude of $\Re\left(h_{11}-h_{22}\right)$ depends on the model of interactions considered. So a new possibility of the verification of models of weak interactions arises.

It also seems, that above results have some meaning when attempts to describe possible deviations from conventional quantum mechanics are made and when possible experimental tests of such a phenomenon and CPTinvariance in the neutral kaons system are considered [35, 36. In such a case a very important role is played by nonzero contributions to $\left(h_{11}-h_{22}\right)$ 35. 36]: The correct description of these deviations and experiments mentioned is impossible without taking into account the results of this Section and the above Sec. 2. This can not be performed within the LOY approach and requires more exact approximations. It seems that the approximation described and exploited in [20] - 22] may be a more effective tool for this purpose.

The above considerations suggest that tests consisting of a comparison of the equality of the decay laws of $\mathrm{K}_{0}$ and $\overline{\mathrm{K}}_{0}$ mesons seem to be the only completely model independent tests for verifying the CPT-invariance in such and similar systems.

Taking into account all the above, it seems that all theories describing the time evolution of the neutral kaon and similar systems by means of the effective Hamiltonian $H_{\|}$governing their time evolution, in which the CPT-invariance of the total system leads to the property (8) for this $H_{\|}$, 
(such as LOY theory [1] — 4] based on the WW approximation), can not lay claim to being the exact and correct description of all aspects of the effects connected with the violation or nonviolation of the $\mathrm{CP}$ - and especially CPT-symmetries. (It occurs probably because of the fact that such theories cannot exactly satisfy unitarity [19] and lead to inconsistencies of CPTsymmetry properties of the $H_{\|}$and the total Hamiltonians $H$ [33]). Also, it seems that results of the experiments with neutral kaons, etc., designed and carried out on the basis of expectations of theories within the WW approximation, such as tests of CPT invariance (at least the results of those in which CPT-invariance or CPT-noninvariance of $H_{\|}$generated by such invariance properties of $H$ were essential), should be revised using other methods than the WW approach.

The most important observation which follows from the results of Sec. 2 (Conclusion 1) and of [16] is the following one: In CPT invariant system Quantum Theory allows simultaneously created at the instant $t_{0}=0$ unstable particles and their antiparticles as particles with the same masses to have slightly different masses for $t>t_{0}$. Thus some matter-antimatter asymmetry can arise in such system, which can have cosmological consequences [37.

\section{A Appendix}

The aim of this Appendix is to calculate the commutator $\left[\Theta, H_{\|}(t)\right]$ discussed in Sec. 2 and to study some of its applications. In order to calculate this commutator it is convenient to express $H_{\|}(t)$ by means of the formula (20), and then to use assumption (25), the definition of $\left[U_{\|}(t)\right]^{-1}$ (19) and the following property

$$
P\left[U_{\|}(t)\right]^{-1}=\left[U_{\|}(t)\right]^{-1} P \equiv P\left[U_{\|}(t)\right]^{-1} P,
$$

which is the consequence of (19). This last observation together with the property (17) means that the identity (18) can be replaced by the following one:

$$
H_{\|} \equiv H_{\|}(t) \equiv i \frac{\partial U_{\|}(t)}{\partial t}\left[U_{\|}(t)\right]^{-1} P
$$

Now one can consider a commutator $\left[\Theta, P\left[U_{\|}(t)\right]^{-1}\right]$. It is the only nontrivial relation, necessary for the calculation of $\left[\Theta, H_{\|}(t)\right]$. Using property (A.1) and definition (19), we find (here the assumption (25) is crucial)

$$
\left[\Theta, P\left[U_{\|}(t)\right]^{-1}\right]=\Theta P\left[U_{\|}(t)\right]^{-1}-P\left[U_{\|}(t)\right]^{-1} \Theta
$$




$$
\begin{aligned}
& =\Theta P\left[U_{\|}(t)\right]^{-1}-P\left[U_{\|}(t)\right]^{-1} P \Theta \\
& =P U_{\|}^{-1}\left(U_{\|} \Theta-\Theta U_{\|}\right) U_{\|}^{-1} \\
& =-P U_{\|}^{-1}\left[\Theta, U_{\|}\right] U_{\|}^{-1} \\
& \equiv-P U_{\|}^{-1} P[\Theta, U] P U_{\|}^{-1} .
\end{aligned}
$$

Properties (A.1) and expression (20) lead to the following formulae

$$
\begin{aligned}
{\left[\Theta, H_{\|}(t)\right] } & =\left[\Theta, P H U P U_{\|}^{-1} P\right] \\
& =[\Theta, P H] U P U_{\|}^{-1}+P H\left[\Theta, U P U_{\|}^{-1}\right] \\
& =P[\Theta, H] U P U_{\|}^{-1} \\
& +P H\left\{[\Theta, U P] U_{\|}^{-1}+U P\left[\Theta, P U_{\|}^{-1}\right]\right\} .
\end{aligned}
$$

All steps in the above formulae and in formulae leading to A.2 have been performed without changing the order of operators appearing in products of type $\Theta H, \Theta U(t)$, etc.. By virtue of the assumption (25) only the order of operators $\Theta$ and $P$ in products $\Theta P$, etc., can be changed when it is necessary.

Now, defining

$$
\mathcal{A}(t) \stackrel{\text { def }}{=} P[\Theta, H] U P U_{\|}^{-1}
$$

(which equals (27) ) and taking into account (A.3), one can obtain formula (26) from (A.4)

$$
\begin{aligned}
{\left[\Theta, H_{\|}(t)\right] } & \equiv \mathcal{A}(t)+P H[\Theta, U] P U_{\|}^{-1} \\
& +P H U P\left\{-U_{\|}^{-1} P[\Theta, U] P U_{\|}^{-1}\right\} \\
& =\mathcal{A}(t)+\mathcal{B}(t)
\end{aligned}
$$

where (see (28))

$$
\mathcal{B}(t)=\left\{P H-P H U P U_{\|}^{-1} P\right\}[\Theta, U] P U_{\|}^{-1},
$$

or (by means of (201) $)$

$$
\mathcal{B}(t) \equiv\left\{P H-H_{\|} P\right\}[\Theta, U] P U_{\|}^{-1},
$$

(i.e., simply (29) ), and due to the properties (21), (22)

$$
\mathcal{B}(t)=\left\{P H Q-V_{\|} P\right\}[\Theta, U] P U_{\|}^{-1},
$$


that is formula (30).

Let us now consider some details of the derivation of the relation (33). Taking into account the properties of the anti-unitary operator $\Theta$ and the CPT-transformation properties of states $\left|K_{0}>,\right| \bar{K}_{0}>$, etc., (see Sec. 2), without any assumptions about the commutator $[\Theta, H]$, one can transform the matrix element $<\mathbf{2}\left|\Theta H_{\|}(t) \Theta^{-1}\right| \mathbf{2}>$ appearing in (133) as follows

$$
\begin{aligned}
<\mathbf{2}\left|\Theta H_{\|}(t) \Theta^{-1}\right| \mathbf{2}>\equiv & <\bar{K}_{0}\left|\Theta H_{\|}(t) \Theta^{-1}\right| \bar{K}_{0}> \\
\equiv & <\Theta K_{0}, \Theta H_{\|}(t) \Theta^{-1} \Theta K_{0}> \\
= & <\Theta^{-1} \Theta H_{\|}(t) \Theta^{-1} K_{0}, \Theta^{-1} \Theta K_{0}> \\
= & <H_{\|}(t) K_{0}, K_{0}> \\
= & <K_{0}, H_{\|}(t) K_{0}>^{*} \\
\equiv & <\mathbf{1}\left|H_{\|}(t)\right| \mathbf{1}>^{*} \equiv h_{11}(t)^{*} .
\end{aligned}
$$

This last relation and the following consequence of (32)

$$
<\mathbf{2}\left|\Theta H_{\|}(t) \Theta^{-1}\right| \mathbf{2}>-<\mathbf{2}\left|H_{\|}(t)\right| \mathbf{2}>\equiv<\mathbf{2}\left|(\mathcal{A}(t)+\mathcal{B}(t)) \Theta^{-1}\right| \mathbf{2}>,
$$

yield

$$
h_{11}(t)^{*}-h_{22}(t)=<\mathbf{2}\left|(\mathcal{A}(t)+\mathcal{B}(t)) \Theta^{-1}\right| \mathbf{2}>,
$$

i.e., the formula (33).

\section{References}

[1] T. D. Lee, R. Oehme and C. N. Yang, Phys. Rev., 106, (1957) 340.

[2] T. D. Lee and C. S. Wu, Annual Review of Nuclear Science, 16, (1966) 471. Ed.: M. K. Gaillard and M. Nikolic, Weak Interactions, (INPN et de Physique des Particules, Paris, 1977); Chapt. 5, Appendix A. S. M. Bilenkij, Particles and nucleus, vol. 1. No 1 (Dubna 1970), p. 227 [in Russian]. P. K. Kabir, The CP-puzzle, Academic Press, New York 1968.

[3] J. W. Cronin, Rev. Mod. Phys. 53, (1981) 373. J. W. Cronin, Acta Phys. Polon., B15, (1984) 419. V. V. Barmin, et al., Nucl. Phys. B247, (1984) 293. L. Lavoura, Ann. Phys. (NY), 207, (1991) 428. C. Buchanan, et al., Phys. Rev. D45, (1992) 4088. C. O. Dib, and R. D. Peccei, Phys. Rev.,D46, (1992) 2265. R. D. Peccei, CP and CPT Violation: Status and Prospects, Preprint UCLA/93/TEP/19, University of California, June 1993. 
[4] E. D. Comins and P. H. Bucksbaum, Weak interactions of Leptons and Quarks, (Cambridge University Press, 1983). T. P. Cheng and L. F. Li, Gauge Theory of Elementary Particle Physics, (Clarendon, Oxford 1984).

[5] K. Kleinkchnet, in: CP Violation, ed. C. Jarlskog, (World Scientific, 1989), p.p. $41-104$.

[6] T. D. Lee, Particle Physics and Introduction to Field Theory, (Harwood Academic Publishers, 1990).

[7] L. I. Bigi and A. I. Sanda, CP Violation, (Cambridge University Press, 2001).

[8] Yu. V. Novozhilov, Introduction to the Theory of Elementary Particles (Nauka, Moskow 1972), (in Russian). W. M. Gibson and B. R. Pollard, Symmetry Principles in Elementary Particle Physics, (Cambridge University Press, 1976).

[9] C. B. Chiu and E. C. G. Sudarshan, Phys. Rev. D 42 (1990) 3712; E. C. G. Sudarshan, C. B. Chiu and G. Bhamathi, Unstable Systems in Generalized Quantum Theory, Preprint DOE-40757-023 and CPP-9323, University of Texas, October 1993.

[10] L. Maiani, in "The Second Daథne Physics Handbook", vol. 1, Eds. L. Maiani, G. Pancheri and N. Paver, SIS - Pubblicazioni, INFN - LNF, Frascati, 1995; pp. $3-26$.

[11] K. Urbanowski and J. Piskorski, Improved Lee, Oehme and Yang approximation, Preprint of the Pedagogical University No WSP-IF 98-51, Zielona Góra, March 1998: physics/9803030; Found. Phys., 30, (2000), 839.

[12] L. P. Horwitz and J. P. Marchand, Helv. Phys. Acta 42 (1969) 801.

[13] K. Urbanowski, Acta Physica Polonica, B 14, (1983), 485.

[14] K. Urbanowski, Phys. Lett. B 313, (1993) 374.

[15] K. Hagiwara et al, Review of Particle Physics, Phys. Rev., D 66, (2002), Part I, No 1-I. 
[16] K. Urbanowski, Physics Letters B 540, (2002), 89; hep-ph/0201272.

[17] L. A. Khalfin, Preprints of the University of Texas at Austin: New Results on the CP-violation problem, (Report DOE-ER40200-211, Feb. 1990); A new CP-violation effect and a new possibility for investigation of $K_{S}^{0}, K_{L}^{0}\left(K^{0}, \bar{K}^{0}\right)$ decay modes, (Report DOE-ER40200-247, Feb. 1991).

[18] L. A. Khalfin, Foundations of Physics, 27, (1997), 1549 and references one can find therein.

[19] P. K. Kabir and A. Pilaftsis, Phys. Rev. A 53, (1996), 66.

[20] K. Urbanowski, Int. J. Mod. Phys. A 7, (1992) 6299. K. Urbanowski, Phys. Lett. A171, (1992) 151.

[21] K. Urbanowski, Int. J. Mod. Phys. A 8, (1993) 3721.

[22] K. Urbanowski, Int. J. Mod. Phys. A 10, (1995) 1151.

[23] K. Urbanowski, Phys. Rev. A 50, (1994) 2847.

[24] K. Urbanowski, CPT transformation properties of the exact effective Hamiltonian for neutral kaon and similar complexes, hep-ph/9803376.

[25] A. Messiah, Quantum Mechanics, vol. 2, (Wiley, New York 1966).

[26] A. Bohm, Quantum Mechanics: Foundations and Applications, 2nd ed., (Springer, New York 1986).

[27] E. P. Wigner, in: "Group Theoretical Concepts and Methods in Elementary Particle Physics", ed.: F. Göresy, (New York 1964).

[28] J. S. Bell and J. Steinberger, in: "Oxford Int. Conf. on Elementary Particles 19/25 September 1965: Proceedings", Eds. T. R. Walsh, A. E. Taylor, R. G. Moorhouse and B. Southworth, (Rutheford High Energy Lab., Chilton, Didicot 1966), pp. $195-222$.

[29] W. Pauli, in: "Niels Bohr and the Developmnet of Physics". ed. W. Pauli (pergamon Press, London, 1955), pp. $30-51$. G. Luders, Ann. Phys. (NY) 2 (1957) 1. . R. Jost, Helv. Phys. Acta 30 (1957) 409.R.F. Streater and A. S. Wightman, "CPT, Spin, Statistics and All That" 
(Benjamin, New York, 1964). N. N. Bogolubov, A. A. Logunov and I. T. Todorov, "Introduction to Axiomatic Field Theory" (Benjamin, New York, 1975).

[30] M. Kobayashi and A. I. Sanda, Phys. Rev. Letters, 69, (1992), 3139.

[31] K. Urbanowski, A new interpretation of one CPT violation test for $K_{0}-$ $\bar{K}_{0}$ system, hep-ph/0202253.

[32] K. Urbanowski, Int. J. Mod. Phys. A 7, (1992) 6299.

[33] K. Urbanowski, Int. J. Mod. Phys. A 13, (1998), 965.

[34] W. Krolikowski and J. Rzewuski, Bull. Acad. Polon. Sci. 4 (1956) 19. W. Krolikowski and J. Rzewuski, Nuovo. Cim. B 25 (1975) 739 and refernces therein.

[35] J. Ellis, J. S. Hagelin, D. V. Nanopoulos and M. Srednicki, Nucl. Phys. B241, (1984) 381. J. Ellis, N. E. Mavromatos and D. V. Nanopoulos, Phys. Lett. B 293, (1992) 142. J. Ellis, J. L. Lopez, N. E. Mavromatos and D. V. Nanopoulos, Phys. Rev. D 53, (1996) 3846.

[36] P. Huet and M. E. Peskin, Nucl. Phys. B 434, (1995) 3. P. Huet, Testing Violation of CPT and Quantum Mechanics in the $K_{0}-\bar{K}_{0}$ system, Preprint: SLAC-Pub-6491, May 1994.

[37] A. D. Sakharov, Pis'ma Zh. Eksp. Teor. Fiz., 5 (1967) 32 [in Russian], [JETP Letters 5 (1967) 24]; G. R. Farrar and M. E. Shaposhnikov, Phys. Rev. Lett. 70 (1993) 2833 and Phys. Rev. D50 (1994) 774; M. B. Gavela, P. Hernandez, J. Orloff and O. Pene, Nucl. Phys. B 430 (1994) 345, 382; K. A. Olive, Bing Bang Baryogenesis, in Proceedings of the 33rd International Winter School on Nuclear and Particle Physics "Matter Under Extreme Conditions" - Schladming (Austria) 1994, Eds. H. Latal and W. Schweiger (Springer, Berlin, 1994), hep-ph/9404352 V. A. Rubakov and M. E. Shaposhnikov, Uspekhi Fizicheskich Nauk, 166, (1966), 493 and hep-ph/9603208; S. Sarkar, Reports on Progres in Physics, 59, (1996), 1493. M. Trodden, Rev. Mod. Phys. 71,(1999), 1463; hep-ph/9803479. A. Riotto and M. Trodden, Ann. Rev. Nucl. Part. Sci., 49,(1999), 35; hep-ph/9901362. A. Riotto, Theories of baryogenesis, Preprint No CERN-TH/98-204, hep-ph/9807454. A. D. Dolgov, Baryogenesis 30 years after, Preprint No TAC-1997-024 - July 
1997, hep-ph/9707419 A. D. Dolgov, Ya. B. Zeldovich, Reviews of Modern Physics, 53, (1981),1. O.Bertolami, Don Colladay, V. A. Kostelecky and R. Potting, Phys. Lett. B 395, (1997), 178. E. W. Kolb and M. S. Turner, The early Universe, Addison-Wesley, 1993. 